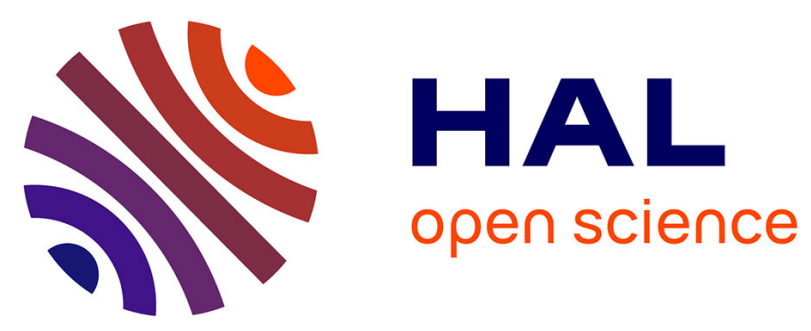

\title{
Contribution du site paléontologique de Lissasfa (Casablanca, Maroc) à une nouvelle interprétation du Mio-Pliocène de la Méseta.
}

Jean-Paul Raynal, David Lefevre, Denis Geraads, Mohssine El Graoui

\section{To cite this version:}

Jean-Paul Raynal, David Lefevre, Denis Geraads, Mohssine El Graoui. Contribution du site paléontologique de Lissasfa (Casablanca, Maroc) à une nouvelle interprétation du Mio-Pliocène de la Méseta.. Comptes rendus de l'Académie des sciences. Série IIa, Sciences de la terre et des planètes, 1999, tome 329, pp.617-622. halshs-00004408

\section{HAL Id: halshs-00004408 \\ https://shs.hal.science/halshs-00004408}

Submitted on 2 Aug 2005

HAL is a multi-disciplinary open access archive for the deposit and dissemination of scientific research documents, whether they are published or not. The documents may come from teaching and research institutions in France or abroad, or from public or private research centers.
L'archive ouverte pluridisciplinaire HAL, est destinée au dépôt et à la diffusion de documents scientifiques de niveau recherche, publiés ou non, émanant des établissements d'enseignement et de recherche français ou étrangers, des laboratoires publics ou privés. 
Titre courant : Lissasfa (Maroc) et le Mio-Pliocène de la Meseta

\title{
Contribution du site paléontologique de Lissasfa (Casablanca, Maroc) à une nouvelle interprétation du Mio-Pliocène de la Méseta.
}

Contribution of the paleontological site of Lissasfa (Casablanca, Morocco) to a new interpretation of the Mio-Pliocene of the Meseta.

\author{
Jean-Paul RAYNAL, David LEFEVRE, Denis GERAADS, Mosshine EL GRAOUI
}

\begin{abstract}
Résumé : la carrière de Lissasfa livre, dans un complexe exokarstique affectant des dépôts supratidaux réputés pliocènes, une microfaune datée vers 5,5 Ma. La base de la longue séquence "plio-pléistocène " de Casablanca est donc d'âge Messinien.
\end{abstract}

Abstract : Lissasfa Quarry presents, in an exokarstic complex affecting reputed Pliocene supratidal deposits, a microfauna approaching 5.5 Myrs in age. The base of the long Plio-Pleistocene sequence of Casablanca is therefore of Messinian age.

Mots-clés : Maroc, Casablanca, microfaune, Mio-Pliocène, Messinien.

Key-Words : Morrocco, Casablanca, microfauna, Mio-Pliocene, Messinian.

Abridged english version :

Between Wadi Mellah to the Northeast and Dar Bou Azza to the Southwest, the hinterland of Casablanca is characterised by a series of large barrier systems sub-parallel to the present beach. Intertidal depositional units, dune formations characteristic of regressive sequences, alteration facies (karsts, palaeosoils) and reworked deposits are associated with each of these morphological units. Stepped from $180 \mathrm{~m}$ above sea level to the present zero, they form the long "quaternary sequence" of Casablanca (Biberson, 1961; Stearns, 1978). The paleontological site of Lissasfa, recently discovered in the most ancient barrier system, now allows us to postulate a minimum age within the Miocene for the base of the sequence.

This barrier system, that rises to $244 \mathrm{~m}$ at Marchich, dominates the plain of Berrechid by about fifty meters. On its north side, Lissasfa quarry, now closed, is situated on the West of P7 road, $14 \mathrm{~km}$ from the coast. Its geology was never described in the literature.

\section{1 - Lithostratigraphy}

In Lissasfa quarry, one observes from the bottom to the top:

- Member 1: A pink facies of altered calcarenite, containing internal molds of gastropods and rare fragments of thick bone. This is truncated by a yellow aeolianite containing large mammal bones and which is characteristic of supratidal surroundings. A few hundred metres to the South, towards Mediouna, intertidal facies rest on a substratum of Cambrian quartzites. This succession characterises a regressive situation responsible for the erection of the Marchich barrier complex.

- Member 2: This is associated with a paleo-shoreline in a micro-cliff cut within Member 1. Its facies characterise an infratidal to supratidal environment. A basal conglomerate is visible resting on a substratum of quartzites at about $155 \mathrm{~m}$ above present sea level. One observes a vertical and lateral transition to a capping aeolianite that conceals the relict shoreline and overlaps deposits of Member 1. Large bones of vertebrates are scattered in the eolianite. Towards the top of Member 2, washout pockets contain a bioclastic sediment formed from the remains of biocalcarenite, rich in bony fragments of micro-mammals and larger vertebrates. 
This vertical and lateral succession of facies characterises a regressive situation. Deposits identified at Lissasfa permit an appreciation of the complexity within the morpho-sedimentary system of the Marchich barriers that can be observed in conjunction with other lithological aspects toward the Southwest in the Koudia al Bahela quarry. We regroup them all in one lithostratigraphic unit that we name the Mediouna Formation.

\section{2 - The fauna of the surface karst}

The faunal remains include a few large Mammals (cf Sivatherium sp.; Rhinocerotidae indet.; Canidae indet.), and a rich microfauna: bat, insectivores and rodents. The Rodent fauna (about 2000 teeth) includes (Geraads, 1998):

Muridae:

- Paraethomys lissasfensis Geraads, 1998. The genus Paraethomys, known from the late Miocene onwards, includes several lineages lasting until the late Pleistocene in North Africa and Spain. This species is distinct from other North African ones, and more akin to Spanish populations (Caracava and Alcoy).

- Mus ique Geraads, 1998. This small mouse displays derived features (long prelobe on M1/, short M3s) which set it apart from later species in the same area.

- Praomys sp.

No other Murid has been discovered, in contrast to several other North African sites of similar age.

Cricetidae:

- Lophiomys maroccanus Aguilar \& Michaux, 1990. Lophiomys, today restricted to Ethiopia, is known in a few Moghrebian and Spanish sites of the Miocene and Pliocene. This species was first described from Khemis near Safi.

- Ruscinomys africanus Geraads, 1998. This genus, previously identified in Moghreb by a few teeth only, is abundantly represented here by a species noticeable by having its third molars less reduced than in other species of the Western Mediterranean.

Gerbillidae:

- Myocricetodon sp. A few teeth recall those of the late Miocene of Spain and Morocco.

- Protatera davidi Geraads, 1998. This species, the most abundant Rodent of Lissasfa, belongs to a late Miocene / early Pliocene group, within which phyletic relationships are still unclear but, once again, its closest relatives are found in Spain.

- Gerbillidae indet.

Ctenodactylidae:

- Irhoudia sp.

Sciuridae:

- Atlantoxerus sp.

With four new species, the Rodent fauna of Lissasfa is too original to be easily correlated with any previously established bio-chronological scale, such as that provided by Coiffait-Martin (1991). The site cannot be older than the late Miocene, because of the occurrence of Mus and Paraethomys, but is not more recent than the early Piocene, because of the occurrence of Myocricetodon, a primitive Ruscinomys, and because of the absence of Golunda.

The evolutionary stages of Paraethomys and Protatera best fit an age close to the MioPliocene boudary. Thus, the age of Lissasfa would be similar to those of Argoub Kemellal in Algeria (Coiffait-Martin, 1991) and Aïn Guettara in Eastern Morocco (Brandy \& Jaeger, 1980). However, these latter sites contain several taxa of European origin (Stephanomys, Apodemus, Cricetus) that are missing at Lissasfa. This may be explained either by several migration events or, more probably, by a bio-geographic differentiation between the coastal plains and the hinterland.

\section{3 - Discussion}

The association of micromammal characteristics from the superficial karst of Lissasfa suggest an age towards the Mio-Pliocene boundary, in the vicinity of $5.5 \mathrm{Ma}$, and therefore fixes a 
minimum age for the Mediouna Formation. These animals also indicate a very open vegetation setting, analogous to the one recognised in the upper Pliocene at Ahl Al Oughlam and related to the global climatic crisis of $2.4 \mathrm{Ma}$.

For Stearns (1978), formations met at Mediouna fit into shell calcarenites of the plain of Berrechid (Pliocene of the Meseta of Lecointre, 1952, 1963 and Gigout, 1956) assigned to the Moghrebien and are related to the Fouaratien of the Rabat region, a stratigraphic unit of the recent Pliocene. Data obtained from the Lissasfa site show that in fact these formations represent the Messinian and that the so-called Pliocene of the Meseta is Miocene in age.

These new interrelationships show a different structural comportment than that previously supposed during the final Miocene and the Pliocene: the region of Rabat was in subsidence during the Messinian and the Middle Pliocene whereas the Casablanca hinterland functioned as a coastal platform before the Messinian.

Besides, contrary to the classic interpretation of other authors, the whole Pliocene event here does not correspond to just one regressive sequence on a platform. The Pliocene deposits are complex morpho-sedimentary units, with cut-and-fill structures and fashioning of beaches that testify to bathymetric variations implying multipleadvances and retreats, interpreted, along with isotopic data, as the consequence of fluctuations of weak ampleness in the oceanic volume on a relatively steady platform.

\section{4 - Conclusion}

The palaeontological data from Lissasfa permits a bio-stratigraphic dating based of the long sequence of Casablanca that begins at the Mio-Pliocene boundary in a regional climate context tending towards aridity, synchronous of a generalised lowering of the marine level: deposits of the Formation of Mediouna are Messinian in age.

Therefore since the final Miocene, the long sequence of Casablanca represents an exceptional register of the global oceanic level variation srelative to the fluctuations of the global glaciation in the high latitudes.

Introduction

Entre l'Oued Mellah au Nord-Est et Dar Bou Azza au Sud-Ouest, l'arrière-pays de Casablanca est caractérisé par un modelé de grands cordons subparallèles au rivage actuel. A chaque unité morphologique sont associés des dépôts intertidaux à supratidaux et dunaires caractéristiques de séquences régressives, des faciès d'altération (karsts, paléosols) et de remaniement. Etagés de $180 \mathrm{~m}$ NGM jusqu'au zéro actuel, ils forment la longue séquence "quaternaire" de Casablanca (Biberson, 1961 ; Stearns, 1978). Le riche gisement paléontologique d'Ahl al Oughlam, situé autour de 2,4 Ma, avait permis d'établir que la partie ancienne de cette séquence, au-delà de 100m NGM, se situait franchement dans le Pliocène (Raynal et al., 1990, Geraads et al. 1998). Le site paléontologique de Lissasfa, découvert récemment dans le système de cordons le plus ancien, montre que le bas de la séquence remonte au moins au début du Pliocène.

Ce système de cordon qui culmine à $244 \mathrm{~m}$ à Marchich, initie le style morpho-sédimentaire caractéristique de l'arrière-pays de Casablanca. II domine d'une cinquantaine de mètres la plaine de Berrechid. Sur sa retombée nord, s'ouvre la carrière de Lissasfa qui appartient au groupe d'excavations situées de part et d'autre de la route P7 joignant Casablanca à Mediouna, à 14 kilomètres environ du littoral actuel $(X=301,5 \quad Y=322,2)$. Cette carrière, aujourd'hui en fin d'exploitation, est située à l'Ouest de la route et n'a jamais été décrite dans la littérature.

Dans le cadre des travaux franco-marocains engagés depuis 1978 à Casablanca, elle a fait l'objet en 1986 d'une première reconnaissance avec repérage de fossiles dispersés (J.P. 
Raynal et J.P. Texier, rapport au Service de I'Archéologie du Maroc le 22 mai 1986). Lors d'une révision conduite en 1995, une abondante microfaune associée à quelques gros ossements fut découverte au sommet de la série stratigraphique visible, dans un complexe continental exokarstique. Des ramassages limités et un examen détaillé du matériel récolté ont confirmé la très grande ancienneté et le caractère tout à fait exceptionnel de ce gisement. Des trouvailles occasionnelles ont confirmé depuis la richesse paléontologique de ce secteur.

1 - Lithostratigraphie

Dans la carrière de Lissasfa, on observe de bas en haut:

-Membre 1: un faciès d'altération de calcarénite, de couleur rose, à pulmonés et à rares fragments de gros ossements, est tronqué par une éolianite. Le contact est planaire subhorizontal. Les dépôts éoliens, de couleur jaune vif, présentent un litage à pendage de 25 à $35^{\circ}$ vers l'ESE et contiennent de gros ossements. Ces faciès caractérisent des milieux supratidaux. Quelques centaines de mètres plus au Sud, vers Médiouna, des faciès intertidaux reposent sur le substrat de quartzites cambriens. Cette succession caractérise une situation régressive responsable de l'édification du complexe de cordons de Marchich.

-Membre 2 : il est associé à un paléo-rivage en micro-falaise façonné aux dépens du Membre 1. Ses faciès caractérisent des milieux infratidaux à supratidaux.

Sa base conglomératique est visible à l'affleurement dans le paléo-rivage et repose sur le substrat de quartzites vers 155 mètres NGM. Elle passe verticalement à une biocalcarénite grossière à lits lumachelliques qui se présente principalement en mégastructures planaires de puissance métrique, à pendage de 2 à $5^{\circ}$ vers l'O-ONO, à litage interne de 5 à $25^{\circ}$ vers l'ONO ou le NN0, entrecoupées d'auges plurimétriques peu creusées $(0,50 \mathrm{~m})$. En lame mince, le faciès est de "type $A$ " (El Graoui, 1994), avec des fragments de lamellibranches à tests aragonitiques ou de calcite magnésienne partiellement dissous. La cimentation précoce est matérialisée par une enveloppe micritique en frange régulière autour des éléments, d'origine marine phréatique de zone infratidale à subtidale. Une cimentation tardive de microsparite et de sparite moyenne indique des phénomènes de dissolution-recristallisation sous l'action de circulations vadoses continentales.

Vers le paléorivage en revanche, la cimentation précoce est matérialisée par une frange irrégulière micritique indicative d'un milieu marin vadose de zone intertidale. Une phase d'apport continentale est matérialisée par un dépôt ferrugineux dans les vides de dissolution intragranulaires; concommitante de la phase de cimentation précoce, elle souligne la croissance des cristaux de la frange sous forme de fins liserés. Une phase de cimentation tardive, microsparitique à sparitique, colmate les vides intergranulaires et résulte de processus de dissolution-recristallisation liés aux circulations vadoses continentales. Des vides vacuolaires développés aux dépens des bioclastes et de la cimentation attestent d'une phase ultime de dissolution du dépôt.

Vers le haut, on passe à une calcarénite à mégalitage planaire oblique vers l'ONO et à microlitage interne à pendage de 25 à $30^{\circ}$ vers l'ENE. En lame mince, le faciès est toujours de "type A " (El Graoui, 1994), relativement riche en micro-organismes dont de nombreux foraminifères. Les bioclastes allongés soulignent la stratification interne du dépôt. La cimentation précoce est représentée par une frange micritique irrégulière autour des bioclastes, indicative d'un milieu marin vadose de zone intertidale. Un second ciment microsparitique à sparitique s'est formé en milieu continental vadose. La sédimentation interne est ferrugineuse et colmate les vides de dissolution intragrains ; elle est reprise par la dissolution et correspond à des apports continentaux intervenus après la cimentation précoce.

On observe un passage vertical et latéral à une éolianite sommitale qui masque la ligne de rivage et recouvre les dépôts du Membre 1. De gros ossements de vertébrés y sont épars. Au sommet du Membre 2, des poches décamétriques contiennent un sédiment bioclastique remanié de biocalcarénites, riche en fragments osseux de micromammifères et de grands vertébrés. En lame mince, on note une intense bioturbation indiquée par de nombreux pores racinaires. La cimentation intergranulaire est microsparitique à sparitique, liée à la redistribution des carbonates par les circulations vadoses. Les bioclastes sont très déstructurés et recristallisés et sont enrobés par un 
matériel ferrugineux également recristallisé. La recristallisation est due aux phases de dissolutionrecristallisation sous l'action des circulations vadoses continentales.

Cette succession verticale et latérale de faciès au sein du Membre 2 caractérise une séquence régressive.

Les dépôts identifiés à Lissasfa permettent d'apprécier la complexité interne du système morphosédimentaire de cordons de Marchich dont d'autres aspects lithologiques peuvent être observés vers le Sud-Ouest dans la carrière de Koudia al Bahela. Nous les regroupons en une seule unité lithostratigraphique que nous nommons "Formation de Médiouna".

\section{2 - La faune du remplissage karstique superficiel}

Les éléments fauniques comportent quelques restes de grands mammifères (cf. Sivatherium sp., Rhinocérotidé indét. et Canidé indét.) et une riche microfaune : chiroptère, insectivores et rongeurs. L'association de rongeurs (environ 2000 dents) comporte selon Geraads (1998) :

Muridae :

- Paraethomys lissasfensis Geraads, 1998. Le genre Paraethomys apparu au Miocène supérieur comporte plusieurs lignées qui perdurent jusqu'au Pléistocène supérieur en Afrique du Nord et en Espagne. L'espèce découverte à Lissasfa se distingue bien de celles connues à la même époque au Maghreb, mais évoque davantage des populations des sites espagnols de Caravaca et Alcoy.

- Mus ique Geraads, 1998. Cette petite souris présente des caractères évolués, comme un prélobe de $\mathrm{M} 1 /$ long et une $\mathrm{M} 3 /$ courte, qui la distinguent bien des formes plus récentes de la même région.

$$
\text { - Praomys sp. }
$$

Aucun autre Muridé n'a été découvert, à la différence de plusieurs autres sites d'âge voisin au Maghreb : Aïn Guettara (Brandy et Jaeger, 1980) et Argoub Kemellal (Coiffait-Martin, 1991).

Cricetidae:

- Lophiomys maroccanus Aguilar et Michaux, 1990. Ce genre, qui ne survit aujourd'hui qu'en Ethiopie, est présent dans quelques gisements maghrébins et espagnols du Miocène et du Pliocène. L'espèce de Lissasfa semble identique à celle de Khémis près de Safi (Aguilar et Michaux, 1990).

- Ruscinomys africanus Geraads, 1998. Ce genre n'était connu au Maghreb que par quelques dents. II est ici abondant et représenté par une espèce remarquable par ses troisièmes molaires moins réduites que chez les formes comparables de Méditerranée occidentale.

Gerbillidae:

- Myocricetodon sp. Quelques dents évoquent les populations du Miocène terminal d'Espagne et du Maroc.

- Protatera davidi Geraads, 1998. Cette espèce, la plus abondante du gisement, appartient à un groupe du Miocène terminal et du début du Pliocène dont les rapports phylétiques sont encore incertains, mais là encore, les plus proches parents de la forme de Lissasfa semblent plutôt devoir être recherchés en Espagne.

- Gerbillidé indet.

- Ctenodactylidae: Irhoudia sp.

- Sciuridae : Atlantoxerus sp.

L'originalité de la faune de Rongeurs du karst superficiel de Lissasfa, qui comprend au moins 4 espèces nouvelles, rend délicate son intégration dans une échelle biochronologique 
existante, telle que celle établie par Coiffait-Martin (1991). Les présences de Mus et de Paraethomys interdisent de repousser le gisement au-delà de la fin du Miocène supérieur, tandis que celles de Myocricetodon, d'un Ruscinomys primitif, l'absence de Golunda, impliquent que le site ne saurait être plus récent que le Pliocène basal. Un âge voisin de la limite Mio-Pliocène est celui qui s'accorde le mieux avec le stade évolutif de Paraethomys et de Protatera. L'âge de Lissasfa serait donc proche de ceux d'Argoub Kemellal en Algérie (Coiffait-Martin, 1991) et d'Aïn Guettara au Maroc oriental (Brandy \& Jaeger, 1980). Cependant, plusieurs taxons d'origine européenne présents dans ces derniers sites (Stephanomys, Apodemus, Cricetus) manquent à Lissasfa, pourtant caractérisé par des influences espagnoles. II faut donc envisager, soit l'existence de phases d'échanges distinctes au Mio-Pliocène, soit, plus vraisemblablement, une différenciation biogéographique entre le littoral marocain et l'arrière-pays.

\section{3 - Discussion}

Les caractères de l'association de micromammifères du karst superficiel de Lissasfa la situent vers la limite mio-pliocène, aux environs de 5,5 Ma, et fixent donc un âge minimum pour la Formation de Mediouna. Elles indiquent en outre un milieu très ouvert.

Pour Stearns (1978), les formations rencontrées à Mediouna sont emboîtées dans les calcarénites à pectinidés de la plaine de Berrechid (Pliocène de la Méseta de Lecointre, 1952, 1963 et Gigout, 1956) attribuées au Moghrébien et sont mises en corrélations avec le Fouaratien de la région de Rabat, unité stratigraphique du Pliocène récent. Les données obtenues sur le site de Lissasfa montrent que ces formations représentent en fait le Messinien et que le Pliocène de la Méseta est d'âge Miocène.

Les formations rencontrées plus au Nord entre 100 et 120 m NGM et emboîtées dans les précédentes étaient censées représenter la base du Pléistocène (Messaoudien ) (Biberson, 1961, Stearns, 1978). Les données obtenues sur le site d'Ahl Al Oughlam montrent qu'elles datent d'un Pliocène antérieur à 2,4 Ma et peuvent donc être mises partiellement en corrélation avec les dépôts “ moghrébiens" de la région de Rabat, d'âge Pliocène moyen (Wernli, 1978, 1979).

Ces nouvelles corrélations montrent un comportement structural différent au cours du Miocène final et du Pliocène : la région de Rabat fonctionne en subsidence au Messinien et au Pliocène moyen alors que l'arrière pays de Casablanca fonctionne déja en plate-forme littorale avant le Messinien.

La phase régressive responsable de la mise en place de la Formation de Mediouna et en particulier de l'édification des grands cordons de Marchich peut être mise en relation avec la commande globale : extension de la calotte antarctique entre 7 et $5 \mathrm{Ma}$ (Shackleton et Kennett, 1975) et abaissement du niveau de l'Océan mondial de l'ordre de 50 m (Berggren et Haq, 1976). L'association faunique du sommet du Membre 2 indique d'ailleurs un couvert végétal très ouvert, conséquence de cette crise d'aridité du Messinien.

En outre, contrairement à l'interprétation classique des auteurs, l'ensemble du dispositif pliocène ne correspond pas à une seule séquence régressive sur une plate-forme. Les dépôts pliocènes sont en effet des unités morpho-sédimentaires complexes, avec emboîtement de formations et façonnage de rivages, qui témoignent de variations bathymétriques impliquant des aller-retours multiples. Le dispositif de plate-forme met à disposition un stock sédimentaire abondant lors des phases de retrait de la mer à commande globale et autorise ainsi la construction de grands systèmes de cordons essentiellement dunaires, sans que cela implique pour autant des changements significatifs du climat régional. L'absence d'étagements multiples peut être interprétée, en accord avec les données isotopiques, comme la conséquence de fluctuations du volume océanique de faible ampleur et de durée différente de celle du modèle pléistocène, sur une plateforme relativement stable. A partir du Pleistocène inférieur en revanche, la diversité et la complexité des enregistrements étagés, emboîtés, voire superposés, entre $90 \mathrm{~m} \mathrm{NGM}$ et le zéro actuel, 
s'explique par un contrôle tectonique combiné avec des variations eustatiques de plus grande ampleur.

\section{4 - Conclusion}

Les données paléontologiques obtenues à Lissasfa permettent le calage biostratigraphique de la base de la longue séquence de Casablanca qui débute à la charnière miopliocène dans un contexte de climat régional à tendance aride synchrone d'un abaissement généralisé du niveau marin : les dépôts de la "Formation de Médiouna " sont d'âge Messinien.

La longue séquence de Casablanca représente donc, dès le Miocène final, un enregistrement exceptionnel des variations du niveau de l'Océan mondial en relation avec les fluctuations de l'englacement planétaire aux hautes latitudes.

Autorisation de publier $n^{\circ}$ 03/98-99 du 15 janvier 1999.

J.P.R., Université de Bordeaux 1, Institut de Préhistoire et de Géologie du Quaternaire, UMR 5808 CNRS, Avenue des Facultés, 33405 Talence Cedex (France) - Mission Littoral (MAE) et équipe Casablanca (INSAP).jpraynal@wanadoo.fr

D.L. Université de Montepellier III, Département de Géographie, BP 5043, 34032 Montpellier Cedex 1 (France) et UMR 9944 CNRS - Mission Littoral (MAE) et équipe Casablanca (INSAP). geophys@smr1.univ-montp3.fr

D.G. CNRS - EP 1781 CNRS, IRESCO, 59 rue Pouchet, 75849 Paris Cedex, Mission Littoral (MAE) et équipe Casablanca (INSAP). dgeraads@compuserve.com

M.E.G., Ministère des Affaires Culturelles, Direction du Patrimoine, Parc National du Patrimoine Rupestre, Marrakech (Maroc) et équipe Casablanca (INSAP).

Remerciements

Nous remercions l'Institut National des Sciences de l'Archéologie et du Patrimoine du Royaume du Maroc, le Ministère des Affaires Etrangères, la Région Aquitaine et la Chaire de Paléoanthropologie et Préhistoire du Collège de France (Pr. Y. Coppens) qui ont participé au financement de ces travaux, F. Amani, R. Bernoussi, A. Khaddouma, L. Magoga, A. Mohib, A. Nader, F.Z. Sbihi-Alaoui et J.P. Texier qui ont participé aux travaux de terrain et $P$. Bindon.

\section{Références bibliographiques}

AGUILAR J.-P. \& MICHAUX J., 1990. Un Lophiomys (Cricetidae, Rodentia) nouveau dans le Pliocène du Maroc; rapport avec les Lophiomyinae fossiles et actuels. Paleontologia i Evolucio, 23: 205-211.

BIBERSON P., 1961. Le cadre paléogéographique de la Préhistoire du Maroc atlantique et le Paléolithique inférieur du Maroc atlantique, Publications du Service des Antiquités du Maroc, Rabat, 1961 , fasc. 16,235 p. et 17,544 p.

BERGGREN W.A. et HAQ B., 1976. The Andalusian stage (Late Miocene) : Biostratigraphy, biochronology and palaeoecology. Palaeogeogr., Palaeoclim., Palaeoecol., 20 : 67-129.

BRANDY L.D. \& JAEGER J.J., 1980. Les échanges de faunes terrestres entre l'Europe et l'Afrique au Messinien. C. R. Acad. Sc. Paris, D, 291: 465-468. 
COIFFAIT-MARTIN B., 1991. Contribution des Rongeurs du Néogène d'Algérie à la biochronologie mammalienne d'Afrique nord-occidentale. Thèse Univ.Nancy-I, 389p.

EL GRAOUI M., 1994. Contribution à l'étude des formations littorales quaternaires de la région de Casablanca (Maroc) : sédimentologie, microfaciès et minéraux lourds. Thèse de l'Université de Bordeaux I, $n^{\circ} 1100,288 p$.

GERAADS D., 1998. Rongeurs du Mio-Pliocène de Lissasfa (Casablanca, Maroc). Geobios, 31 (2) : 229-245.

GERAADS D., AMANI F., RAYNAL J.P., SBIHI-ALAOUI F.Z, 1998. La faune de Mammifères du Pliocène terminal d'Ahl Al Oughlam, Casablanca, Maroc. C.R. Acad. Sci. Paris, 326 : 671-676.

GIGOUT M., 1956. Recherches sur le Pliocène et le Quaternaire atlantiques marocains. Travaux de l'Institut Scientifique Chérifien, Série Géologie et Géographie Physique, n 5, 94 p.

LECOINTRE G.,1952. Recherches sur le Néogène et le Quaternaire marins de la côte atlantique du Maroc. Notes et Mémoires du Service Géologique du Maroc, $n^{\circ}$ 99, t.1 : stratigraphie, 198 p. et t.2 : paléontologie, $172 \mathrm{p}$.

LECOINTRE G.,1963. Recherches sur le Néogène et le Quaternaire marins de la côte atlantique du Maroc. Tome 3 : les acquisitions nouvelles durant la période de 1952 à 1962. Notes et Mémoires du Service Géologique du Maroc, $n^{\circ} 174,76$ p.

RAYNAL J.P., TEXIER J.P., GERAADS D., SBIHI-ALAOUI F.Z., 1990. Un nouveau gisement paléontologique plio-pléistocène en Afrique du Nord: Ahl Al Oughlam (ancienne carrière Deprez) à Casablanca (Maroc). C.R. Acad. Sci. Paris, série II, $310: 315-320$.

TEXIER J.P., RAYNAL J.P., LEFEVRE D., 1985. Nouvelles propositions pour un cadre chronologique raisonné du Quaternaire marocain. C.R. Acad. Sci. Paris, série II, 301 : p. 183-188.

SHACKLETON N.J. \& J.P. KENNETT J.P., 1975. Late Cenozoic oxygen and carbon isotope changes at DSDP site 284 : Implications for glacial history of the northern hemisphere and antarctica. Init. Rep. Deep-Sea Drill. Proj., 29 : 801-807.

STEARNS C.E., 1978. Plio-pleistocene emergence of the moroccan Meseta. Geological Society of America Bulletin, 89, p. 1630-1644.

WERNLI R., 1978. La base du Moghrébien est d'âge Pliocène moyen (Zone à G. crassaformis) dans la Mamora (Maroc). Arch. Sc. Genève , 31 (2) :129-132.

WERNLI R., 1979. Le Pliocène de la Mamora (Maroc) : stratigraphie et foraminifères planctoniques. Eclogae. Geol. Helv. 72 (1) : 110-143. 